\title{
Nanomateriales para la valorización de compuestos provenientes de fuentes renovables
}

\section{Nanomaterials for the recovery of compounds from renewable}

\section{sources}

Presentación: 22/11/2019

\section{Doctorando:}

\section{Viola, Belén Melisa}

Centro de Investigación y Tecnología Química (CITeQ)- Universidad Tecnológica Nacional, Facultad Regional Córdoba (UTN-frc). violabelen@gmail.com

\section{Director/es:}

\section{Directora: Casuscelli, Sandra G. Co-directoras: Cánepa, Analía L., Eimer, Griselda A.}

\begin{abstract}
Resumen
Se estudió la funcionalización del alcohol trans-2-hexen-1-ol proveniente de una fuente natural con el objetivo de obtener productos de mayor valor agregado mediante la aplicación de catálisis heterogénea. Para ello, se emplearon arcillas naturales procedentes de la provincia de San Juan, Argentina, que fueron modificadas químicamente con el fin de evaluar su actividad catalítica en la reacción de oxidación del alcohol trans-2-hexen-1-ol. Así, se les realizó un proceso de intercambio iónico y posterior pilareado con titanio. Se obtuvieron sólidos que fueron caracterizados por DRX e isotermas de adsorción-desorción de $\mathrm{N}_{2}$. Para llevar a cabo la reacción, se empleó peróxido de hidrógeno como oxidante, acetonitrilo como solvente y la temperatura se mantuvo en $70{ }^{\circ} \mathrm{C}$. Los resultados mostraron que al pilarear el sólido, la conversión del sustrato se incrementó y, a pesar de que la selectividad hacia el 2,3-epoxihexanol se favoreció, se obtuvieron altos rendimientos de reacción al 2-hexenal (12\%). Luego se varió la relación sustrato/oxidante. Al usar una relación molar igual a 1, la selectividad al epóxido disminuyó favoreciéndose la formación del 2-hexenal y alcanzando un rendimiento de reacción cercano al 20\%.
\end{abstract}

Palabras claves: Arcillas pilareadas, Oxidación, trans-2-hexen-1-ol.

\begin{abstract}
The functionalization of trans-2-hexen-1-ol alcohol from a natural source was studied in order to obtain high value-added products through the application of heterogeneous catalysis. For this, natural clays from the province of San Juan, Argentina were used, which were chemically modified in order to evaluate their catalytic activity in the oxidation reaction of the trans-2-hexen-1-ol alcohol. Thus, an ion exchange process and subsequent pilareado with titanium were performed. Solids were obtained which were characterized by XRD and adsorption-desorption isotherms of $\mathrm{N}_{2}$. Hydrogen peroxide was used as oxidant, acetonitrile as solvent and the reaction temperature was maintained at $70{ }^{\circ} \mathrm{C}$. The results showed that when the solid were pillared, the substrate conversion increased and although the selectivity towards 2,3-epoxyhexanol was favored, high reaction yields for 2-hexenal were obtained $(12 \%)$. Then, the substrate/oxidant molar ratio was varied. When using a molar ratio equal to 1 , it was found that epoxide selectivity decreased, favoring the formation of 2 -hexenal with a yield close to $20 \%$.
\end{abstract}

Keywords: Pillared clays, Oxidation, trans-2-hexen-1-ol. 


\section{Introducción}

El (Z)-2-hexen-1-ol es un compuesto orgánico volátil que puede extraerse de la naturaleza al encontrarse presente en las plantas. Particularmente, se halla en abundancia en las hojas y en las bayas de las uvas y en menor proporción en arándanos [1]. La reacción de oxidación de este alcohol tiene importancia industrial ya que los productos obtenidos, sea por la oxidación del grupo alcohol, epoxidación de la olefina u oxidaciones sucesivas de los mismos, dan lugar a la formación de intermediarios de interés agroquímico, farmacéutico y para la industria química en general $[2,3]$.

Uno de los principales desafíos de la química contemporánea es el desarrollo de nuevas tecnologías alternativas que sean limpias, seguras y amigables con el medio ambiente. Muchos de esos objetivos se lograron mediante el empleo de catalizadores heterogéneos [4]. Así, la catálisis heterogénea ha contribuido al desarrollo de numerosos procesos de química fina, entre ellos, la oxidación selectiva de compuestos orgánicos mejorando el proceso de producción así como la eliminación de productos indeseables.

Las arcillas pilareadas son materiales cristalinos formados por silicatos estratificados que pueden presentar un arreglo tetraédrico y octaédrico de capas. Las montmorillonitas, con un arreglo formado por una capa octaédrica entre dos capas tetraédricas, presentan cationes en su intercapa que pueden ser intercambiados por cationes o policationes voluminosos [5]. Si estos materiales son sometidos a un tratamiento térmico, esas especies policatiónicas se transforman en pilares rígidos y esto da como resultado la formación de sólidos con elevada área específica, alta porosidad, propiedades ácidas y buena estabilidad térmica. Los materiales así obtenidos, conocidos como arcillas pilareadas, ofrecen un amplio campo de aplicación en procesos catalíticos [6].

Por otro lado, además de emplear catalizadores naturales para oxidar moléculas orgánicas, es importante utilizar oxidantes ambientalmente amigables. El peróxido de hidrógeno $\left(\mathrm{H}_{2} \mathrm{O}_{2}\right)$ tiene ventajas por su valor económico, disponibilidad y por tener un alto contenido de oxígeno activo; además, sólo da agua y oxígeno como subproductos de reacción.

Si bien el plan de tesis asignado abarca múltiples nanomateriales y sustratos naturales, en este trabajo se presentan los resultados obtenidos en la reacción de oxidación del alcohol trans-2-hexen-1-ol en fase líquida utilizando $\mathrm{H}_{2} \mathrm{O}_{2}$ como oxidante y arcillas naturales como catalizadores (Esquema 1). De manera general, los epóxidos son utilizados como intermediarios estratégicos y particularmente 2-hexenal es empleado como fumigante natural y controlador de plagas [7-8].

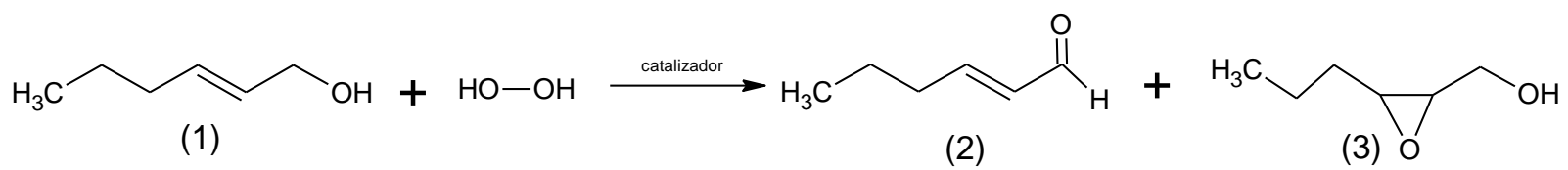

Esquema 1. Productos obtenidos por la oxidación de trans-2-hexen-1-ol: (1) trans-2-hexen-1-ol, (2) 2-hexenal, (3) 2,3-epoxihexanol.

\section{Materiales y Métodos}

Síntesis de catalizadores

Para modificar químicamente las arcillas naturales, se realizó un proceso de intercambio iónico con el fin de intercambiar sus cationes por $\mathrm{Na}^{+}$, obteniéndose la montmorillonita sódica denominada $\mathrm{Na}$-mont. Posteriormente, se realizó el proceso de pilareado donde se adicionó gota a gota a 0,5 L de una suspensión de $8 \mathrm{~g} / \mathrm{L}$ de Na-mont, la solución pilareante. Esta solución se preparó con Ti $\left(\mathrm{OC}_{4} \mathrm{H}_{9}\right)_{4}$ (Fluka) y $\mathrm{HCl} 6 \mathrm{M}$ en una relación molar $\mathrm{H}^{+} / \mathrm{Ti}=2$. La cantidad de solución pilareante agregada fue la necesaria para alcanzar una concentración final de $10 \mathrm{mmol} \mathrm{de} \mathrm{Ti/g}$ de arcilla. Luego del agregado de esta solución, la suspensión se agitó por $12 \mathrm{~h}$ y la fracción sólida se separó por centrifugación. Posteriormente, el sólido se secó y calcinó a $450{ }^{\circ} \mathrm{C}$ durante $2 \mathrm{~h}$, obteniéndose la denominada Ti-PILC.

\section{Caracterización fisicoquímica}

Todos los materiales fueron caracterizados mediante Difracción de Rayos X (DRX) utilizando un difractómetro D8 Advance BRUKER con radiación CuK $\alpha(\lambda=1.5418 \AA ̊) 2 \theta$ entre $2^{\circ}$ y $70^{\circ}$.

Para determinar el área específica y el volumen de poros y microporos, se efectuaron isotermas de absorción y desorción de $\mathrm{N}_{2}$ a $-196{ }^{\circ} \mathrm{C}$ en un equipo Gemini V2.00 Micromeritis. 


\section{Evaluación catalítica}

Las reacciones de oxidación se llevaron a cabo en un reactor batch de vidrio de $10 \mathrm{~mL}$ de capacidad bajo agitación magnética vigorosa. Se empleó trans-2-hexen-1-ol como sustrato (Sigma Aldrich 98\%), peróxido de hidrógeno como oxidante (30\% p/p, Cicarelli) y acetonitrilo como solvente (Sintorgan 99,5\%). A su vez, se trabajó con una relación molar solvente/sustrato igual a $10 \mathrm{y}$ la cantidad de catalizador empleada fue $0,063 \mathrm{~g}$.

El reactor fue sumergido en un baño termostatizado a $70{ }^{\circ} \mathrm{C}$ conectado a un condensador de reflujo. Para evitar la evaporación de la mezcla de reacción se empleó un septum de silicona ubicado en la boca lateral del reactor. Sin apertura del mismo, a través de la boca lateral, se extrajeron muestras a distintos tiempos de reacción, considerando como tiempo cero a la muestra extraída previa a la adición del catalizador en el medio de reacción. Cada muestra fue filtrada y analizada mediante cromatografía gaseosa empleando un cromatógrafo AGILENT 7820 A equipado con una columna HP-1 (30 m de longitud y 0,53 mm de diámetro) y un detector FID. El porcentaje de cada componente en las muestras de reacción fue calculado usando el método de normalización de área empleando factores de respuesta. Además, las muestras fueron analizadas por CG-masa en un cromatógrafo gaseoso con doble detector de masas (TRIPLE CUADRUPOLO) GC-MS/MS Thermo Scientific modelo TSQ 8000 EVO, equipado con inyector automático.

La conversión del alcohol, las selectividades y rendimientos para cada producto obtenido, fueron calculados mediante las ecuaciones 1,2 y 3 respectivamente:

$$
\begin{aligned}
& \text { Conversión }(\%)=100 * \frac{\text { mmoles de productos oxidados }}{\text { mmoles iniciales de reactivo }} \\
& \text { Selectividad }(\%)=100 * \frac{\text { mmoles de producto deseado }}{\text { mmoles totales de productos }} \\
& \text { Rendimiento }(\%)=100 * \frac{\text { mmoles de producto }}{\text { mmoles iniciales de reactivo }}
\end{aligned}
$$

\section{Resultados}

\section{Caracterización fisicoquímica}

En la Figura 1 se observa el patrón de DRX a bajo ángulo, comprendido entre $0^{\circ}$ y $25^{\circ}$. Con los picos característicos de cada estructura analizada y mediante el empleo de la Ley de Bragg, se calcularon los espaciamientos basales de los sólidos analizados (Tabla 1). Como puede observarse en la tabla, al incorporarse el titanio en la matriz arcillosa, se produce un incremento en el espaciamiento basal respecto de la montmorillonita sódica. Esto se debe a que las especies policatiónicas incorporadas en la estructura expanden las capas de las arcillas. En la Figura 2 se presentan los patrones de difracción a alto ángulo (entre $24^{\circ}$ y $70^{\circ}$ ) donde puede apreciarse que las arcillas presentan un pico en $26,7^{\circ}$ que se atribuye al $\mathrm{SiO}_{2}$ (cuarzo). La intensidad de este pico disminuye notablemente luego del proceso de pilareado [9]. A su vez, un nuevo pico se observa en la arcilla pilareada que puede atribuirse $\mathrm{al}^{\mathrm{TiO}_{2}}$ formado en su fase anatasa $\left(2 \theta \approx 25,2^{\circ}\right)$.

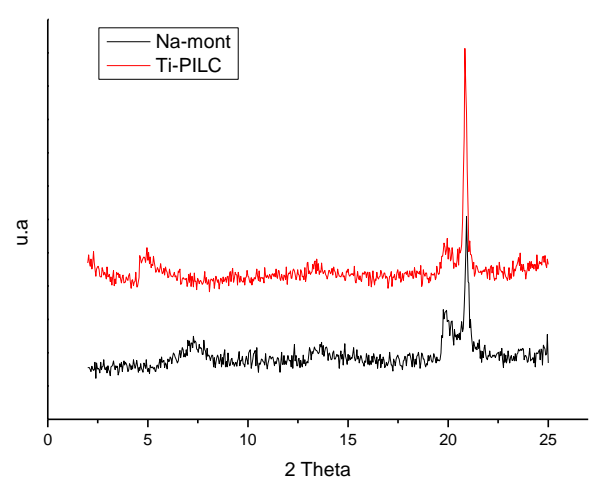

Figura 1. Espectros de DRX a bajo ángulo.

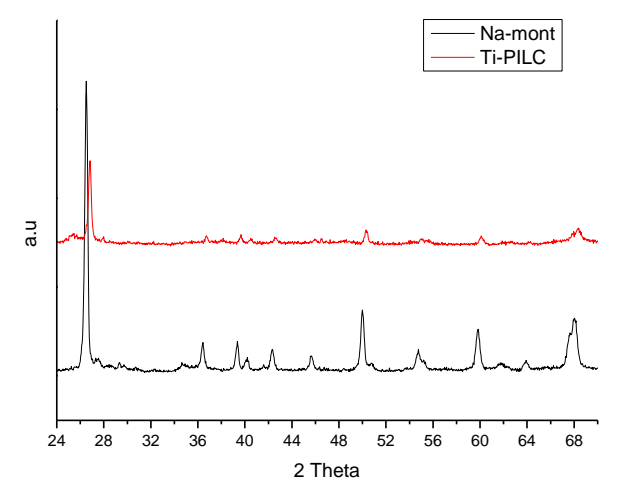

Figura 2. Espectros de DRX a alto ángulo. 
Tabla 1: Propiedades texturales de los catalizadores preparados.

\begin{tabular}{|c|c|c|c|c|}
\hline Catalizador & $\mathbf{d}_{001} \AA$ & $\mathbf{A}_{\text {BET }}\left(\mathbf{m}^{2} / \mathbf{g}\right)^{\mathbf{a}}$ & $\mathbf{V}_{\mathbf{t p b}}\left(\mathbf{c m}^{\mathbf{3}} / \mathbf{g}\right)^{\mathbf{b}}$ & $\mathbf{V}_{\text {Hpc }}\left(\mathbf{c m}^{\mathbf{3}} / \mathbf{g}\right)^{\mathbf{c}}$ \\
\hline Na-mont & 12,27 & 31 & 0,055 & 0,004 \\
\hline Ti-PILC & 17,00 & 105 & 0,131 & 0,003 \\
\hline
\end{tabular}

a Área específica calculada por las isotermas de adsorción de nitrógeno utilizando la ecuación de BET.

b Volumen total de poros calculado a partir de la isoterma de adsorción de nitrógeno.

c Volumen de microporos calculado a partir de la isoterma de adsorción de nitrógeno

Por otra parte, los resultados obtenidos a partir de las isotermas de absorción-desorción de $\mathrm{N}_{2}$ muestran que tanto la superficie específica como la porosidad de Ti-PILC son superiores a las del material de partida (Na-mont). Este hecho refleja la formación de los pilares que expanden las capas de las arcillas.

\section{Evaluación catalítica}

En la Tabla 2 se presentan los resultados obtenidos luego de $5 \mathrm{~h}$ de reacción para la arcilla sódica y la arcilla pilareada. Al utilizar la arcilla sódica se logró una conversión de trans-2-hexen-1-ol de 4,22\% que luego pudo incrementarse a $17,51 \%$ al aplicar el segundo material estudiado. Con estos resultados puede visualizarse la mejora en la actividad catalítica generada por la presencia del pilar de titanio en la estructura.

Los productos de reacción identificados fueron 2,3-epoxihexanol y 2-hexenal, cuyo mecanismo de reacción está bajo estudio.

Tabla 2: Oxidación catalítica de trans-2-hexen-1-ol a 5 h de reacción.

\begin{tabular}{|c|c|c|c|c|c|}
\hline \multirow{2}{*}{ Catalizador } & $\begin{array}{c}\text { Conversión de } \\
\text { alcohol } \\
\end{array}$ & \multicolumn{2}{|c|}{ Selectividad (\%) } & \multicolumn{2}{c|}{ Rendimiento (\%) } \\
\cline { 3 - 5 } & \% molar) & Epóxido & Aldehído & Epóxido & Aldehído \\
\hline Na-mont & 4,22 & 9,33 & 90,67 & 0,39 & 3,83 \\
\hline Ti-PILCa & 17,51 & 27,77 & 72,23 & 4,86 & 12,65 \\
\hline
\end{tabular}

a Reacciones realizadas empleando una relación molar de trans-2-hexen-1-ol/ $\mathrm{H}_{2} \mathrm{O}_{2}=2$.

Posteriormente, se evaluó el efecto de la concentración de oxidante en la conversión. Para ello, se trabajó con la Ti-PILC siendo ésta la que presentó las mayores conversiones y rendimientos de reacción. Para llevar adelante este análisis, se modificaron las relaciones molares de sustrato/oxidante. En la Tabla 3 se indican los resultados obtenidos.

Tabla 3: Oxidación catalítica de trans-2-hexen-1-ol a 5 h de reacción empleando Ti-PILC.

\begin{tabular}{|c|c|c|c|c|c|}
\hline \multirow{2}{*}{$\begin{array}{c}\text { Relaciones } \\
\text { molares }\end{array}$} & \multirow{2}{*}{$\begin{array}{c}\text { Conversión de } \\
\text { alcohol }\end{array}$} & \multicolumn{2}{|c|}{ Selectividad (\%) } & \multicolumn{2}{c|}{ Rendimiento (\%) } \\
\cline { 4 - 6 } alcohol/oxidante & (\% molar) & Epóxido & Aldehído & Epóxido & Aldehído \\
\hline $4 / 1$ & 6,32 & 42,14 & 57,86 & 2,66 & 3,66 \\
\hline $2 / 1$ & 17,51 & 27,77 & 72,23 & 4,86 & 12,64 \\
\hline $1 / 1$ & 27,28 & 26,82 & 73,18 & 7,45 & 20,33 \\
\hline
\end{tabular}

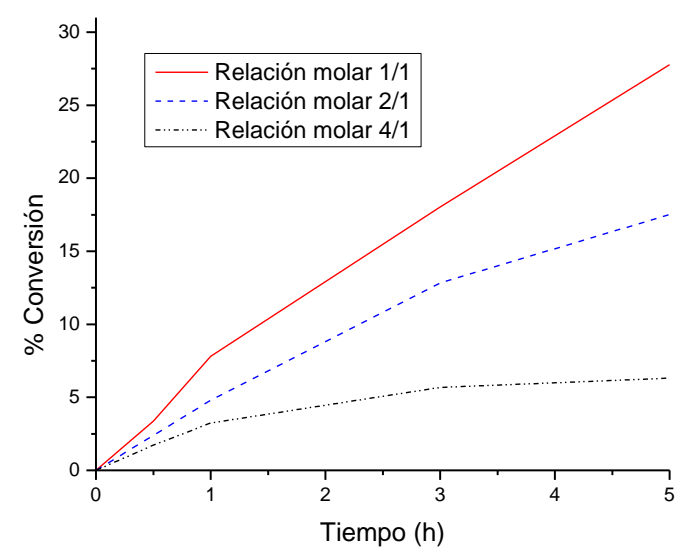

Figura 3. Conversión en función del tiempo.
Como puede observarse, al incrementarse la concentración de oxidante en el medio de reacción, también se incrementa la conversión del trans-2-hexen-1-ol y la selectividad hacia el aldehído 2-hexenal. Asimismo, se logra mejorar el rendimiento de la reacción alcanzando su máximo valor de 20,33\% para una relación molar 1/1. En la Figura 3 se presenta la conversión del alcohol en función del tiempo para las tres relaciones molares estudiadas. En dicha figura puede observarse que la conversión para la relación $1 / 1$ es siempre superior a las otras dos analizadas, dando cuenta de la mejor actividad catalítica obtenida bajo estas condiciones operativas. 


\section{Conclusión}

Se logró caracterizar y pilarear una arcilla sódica empleando titanio. La actividad catalítica de las arcillas fue evaluada en la oxidación del trans-2-hexen-1-ol con $\mathrm{H}_{2} \mathrm{O}_{2}$. Se obtuvo una buena conversión del alcohol con una elevada selectividad al 2-hexenal con la arcilla pilareada (Ti-PILC). Finalmente, aplicando una relación molar 1:1 de sustrato/oxidante se favoreció la conversión del alcohol, incrementándose la selectividad hacia el aldehído. Así, se logró obtener un rendimiento de reacción superior al $20 \%$.

\section{Referencias}

[1] Watkins P., Wijesundera C. (2006). Application of zNosetrade mark for the analysis of selected grape aroma compounds. Talanta, 70, 595-601. doi: 10.1016/j.talanta.2006.01.032.

[2] Lutz J. T. Jr., Kirk-Othmer, M. Grayson, D. Eckroth, G. J. Bushey, C. I. Eastman, A. Klingsberg, L. Spiro. (1980). Encyclopedia of Chemical Technology, 9, 251-266.

[3] Sienel G., Rieth R., Rowbottom K. T., Ullmann's Encyclopedia of Industrial Chemistry, Epoxides, John Wiley\&Sons, 2008.

[4] A. Wróblewska, J. Wajzberg, A. Fajdek, E. Milchert; J. Hazard. Mater. 163 (2009) 1303-1309.

[5] R. S. Varma. Clay and clay-supported reagents in organic synthesis. (2002). Tetrahedron, 58, 1235-1255.

[6] J. Arfaoui, L. Khalfallah Boudali, A.Ghorbel. (2010). Catalytic epoxidation of allylic alcohol (E)-2-Hexen-1-ol over vanadium supported on unsulfated and sulfated titanium pillared montmorillonite catalysts: Effect of sulfate groups and vanadium loading. Applied Clay Science, 48, 171-178.

[7] Fiorella Neri, Marta Mari, Anna Maria Menniti, Stefano Brigati, Paolo Bertolini. (2006). Control of Penicillium expansum in pears and apples by trans-2-hexenal vapours. Postharvest Biology and Technology, 41, 101-108. 10.1016/j.postharvbio.2006.02.005

[8] Weibin M., Luling Z., Yanli X. Inhibitory effect of (E)-2-hexenal as a potential natural fumigant on Aspergillus flavus in stored peanut seeds. Industrial Crops \& Products, 107, 206-210. 10.1016/j.indcrop.2017.05.051.

[9] J. Chen, X. Liu, G. Li, X. Nie, T. An, S. Zhang, H. Zhao. (2011). Synthesis and characterization of novel SiO2 and TiO2 co-pillared montmorillonite composite for adsorption and photocatalytic degradation of hydrophobic organic pollutants in water. Catalisys Today, 164, 364-369. 10.1016/j.cattod.2010.11.014. 